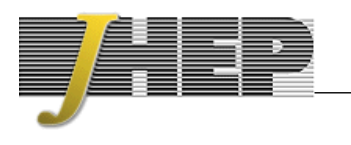

\title{
Topological fermion condensates from anomalies
}

\author{
Simon Catterall, Jack Laiho and Judah Unmuth-Yockey \\ Department of Physics, Syracuse University, \\ Syracuse, NY 13244, U.S.A. \\ E-mail: smcatter@syr.edu, jwlaiho@syr.edu, jfunmuth@syr.edu
}

\begin{abstract}
We show that a class of fermion theory formulated on a compact, curved manifold will generate a condensate whose magnitude is determined only by the volume and Euler characteristic of the space. The construction requires that the fermions be treated as Kähler-Dirac fields and the condensate arises from an anomaly associated with a U(1) global symmetry which is subsequently broken to a discrete subgroup. Remarkably the anomaly survives under discretization of the space which allows us to compute the condensate on an arbitrary triangulation. The results, being topological in character, should hold in a wide range of gravitationally coupled fermion theories both classical and quantum.
\end{abstract}

KeYwords: Lattice Models of Gravity, Lattice Quantum Field Theory, Nonperturbative Effects

ArXiv EPrint: 1806.07845 


\section{Contents}

1 Introduction $\quad 1$

2 Kähler-Dirac fermions in the continuum 1

3 Kähler-Dirac fermions on the lattice 3

4 Condensates 5

5 Numerical results $\quad 6$

6 Conclusions $\quad 8$

\section{Introduction}

Kähler-Dirac fermions have been proposed as an alternative to Dirac fermions in [1] and play an important role in certain supersymmetric field theories where they have been used to construct lattice theories with exact supersymmetry [2]. They also bear a close connection to staggered fermions on regular hypercubic lattices [1, 3].

In this paper we will show that Kähler-Dirac fermions possess another important property; on compact even dimensional spaces with non-zero Euler character they exhibit an anomaly which breaks an exact U(1) symmetry and generates a non-zero fermion condensate.

Rather remarkably this anomaly can be reproduced exactly in a discrete version of the theory, a result which is tied to the structure of the Kähler-Dirac operator and homology theory. We use this fact to compute the anomaly and condensate directly on an arbitrary triangulation.

\section{Kähler-Dirac fermions in the continuum}

Kähler-Dirac fermions naturally arise on taking the square root of the Laplacian operator written in the language of exterior derivatives. Consider the anti-hermitian operator $K=d-d^{\dagger}$ with $d^{\dagger}$ the adjoint of $d$ and $d^{2}=\left(d^{\dagger}\right)^{2}=0$. Clearly $K^{2}=-d d^{\dagger}-d^{\dagger} d=\square$. Following Dirac a natural equation for fermions is then given by

$$
(K-m) \Omega=0
$$

where $\Omega=\left(\omega_{0}, \omega_{1}, \ldots \omega_{p}, \omega_{D}\right)$ is a collection of $p$-forms (antisymmetric tensors). This is the Kähler-Dirac equation and was first proposed as an equation describing fermions in [4]. 
The action of $d$ and $d^{\dagger}$ on these component forms is given by [1]

$$
\begin{aligned}
d \Omega & =\left(0, \partial_{\mu} \omega, \partial_{\mu_{1}} \omega_{\mu_{2}}-\partial_{\mu_{2}} \omega_{\mu_{1}}, \ldots, \sum_{\text {perms } \pi}(-1)^{\pi} \partial_{\mu_{1}} \omega_{\mu_{2} \ldots \mu_{D}}\right) \\
-d^{\dagger} \Omega & =\left(\omega^{\nu}, \omega_{\mu}^{\nu}, \ldots, \omega_{\mu_{1}, \ldots, \mu_{D-1}}^{\nu}, 0\right)_{; \nu} .
\end{aligned}
$$

An inner product of two such Kähler-Dirac fields is then given by

$$
[A, B]=\int d^{D} x \sqrt{g} \sum_{p} \frac{1}{p !} a^{\mu_{1} \ldots \mu_{D}} b_{\mu_{1} \ldots \mu_{D}}
$$

Equation (2.1) can be obtained by variation of the action

$$
S_{\mathrm{KD}}=\int d^{D} x \sqrt{g}[\bar{\Omega}(K-m) \Omega]
$$

where $\bar{\Omega}$ is an independent (in Euclidean space) Kähler-Dirac field. From the Kähler-Dirac field $\Omega$ one can build a matrix $\Psi$ using the Clifford algebra of gamma matrices

$$
\Psi=\sum_{p=0}^{D} \frac{1}{p !} \gamma^{\mu_{1} \ldots \mu_{p}} \omega_{\mu_{1} \ldots \mu_{p}} .
$$

In flat space it is straightforward to show that this fermionic matrix satisfies the usual Dirac equation

$$
\left(\gamma^{\mu} \partial_{\mu}-m\right) \Psi=0
$$

and describes $2^{D / 2}$ degenerate Dirac spinors. Remarkably, eq. (2.1) holds on any smooth manifold so that the coupling of Kähler-Dirac fields to gravity is somewhat different from that of Dirac fermions. This difference can be made manifest if one introduces vielbeins $\gamma_{\mu}=e_{\mu}^{a} \gamma^{a}$ in eq. (2.6) and replaces the derivative in eq. (2.1) with an appropriate covariant derivative containing the spin connection $\omega_{\mu}^{a b}$. This yields a equation which couples the four spinors encountered in flat space through gravitational interactions. It is easily seen that this is not (four copies of) the usual Dirac equation in curved space. In this paper we will exploit the fact that Kähler-Dirac fermions do not require the introduction of this extra spinor structure and work directly with eq. (2.1).

Consider the linear operator $\Gamma$ which transforms the $p$-form fields according to

$$
\Gamma: \quad \omega_{p} \rightarrow(-1)^{p} \omega_{p}
$$

It is straightforward to verify that this operator anticommutes with the Kähler-Dirac operator $\{K, \Gamma\}=0$. This in turn allows us to define a $\mathrm{U}(1)$ symmetry of the massless Kähler-Dirac action

$$
\begin{aligned}
& \Omega \rightarrow e^{i \theta \Gamma} \Omega \\
& \bar{\Omega} \rightarrow \bar{\Omega} e^{i \theta \Gamma} .
\end{aligned}
$$


This symmetry, which is the analog of chiral symmetry for the Dirac theory, ensures that any perturbative mass renormalization of the theory is multiplicative in the bare fermion mass on any background space. It also guarantees that non-zero eigenvalues of $K$ come in pairs, $\pm \lambda_{n}$, with corresponding eigenvectors $\phi_{n}$ and $\Gamma \phi_{n}$ where

$$
K \phi_{n}=\lambda_{n} \phi_{n}
$$

Because $K$ is anti-Hermitian, the eigenvalues are purely imaginary. Of course the symmetry will be broken or anomalous if the fermion measure is not invariant under $\Gamma$. Performing such a transformation yields a Jacobian $J=\operatorname{det}\left(e^{2 i \Gamma \theta}\right)$. Thus the existence of the anomaly depends on a non-vanishing trace for $\Gamma$

$$
\operatorname{Tr} \Gamma=\sum_{n}\left\langle\phi_{n}|\Gamma| \phi_{n}\right\rangle=n_{+}-n_{-}
$$

where the states with non zero eigenvalue are orthogonal and do not contribute while $n_{+}$ and $n_{-}$denote the number of zero modes with eigenvalue \pm 1 respectively. ${ }^{1}$ All of this discussion mirrors the usual arguments given for chiral fermions ${ }^{2}$ and a derivation of the anomaly can be gotten in the continuum after the theory is suitably regulated. However, in this case a simpler method is available. The Kähler-Dirac operator can be discretized using a triangulation of the space in such a way as to preserve the anomaly exactly.

\section{Kähler-Dirac fermions on the lattice}

A triangulation of a $D$-dimensional manifold is constructed by gluing together $D$-simplices. A $p$-simplex consists of $(p+1)$ vertices with every vertex connected to every other vertex. We will restrict our discussion to triangulations in which each $D$-simplex is equilateral with edge length $a$. Such triangulations appear in so-called dynamical triangulation models of quantum gravity $[5,6]$. It is straightforward to generalize this discussion to triangulations with variable edge lengths.

A $p$-simplex contains $(p+1)$ boundary components which are $(p-1)$-simplices. The simplest triangulations or simplicial complexes are constructed by gluing $D$-simplices together along their faces with each face common to two and only two $D$-simplices. The possible vertex labelings of a given $D$-simplex fall into two categories according to whether they consist of an odd or even permutation of the ordered list of vertices. These two categories are called orientations. To define the Kähler-Dirac operator requires that the triangulation be oriented which means that the orientation of each $D$-simplex be chosen in such a way that a given face is held with opposite orientations in the two $D$-simplices that contain it.

There is a natural mapping of differential forms on a continuum manifold to such an oriented discrete triangulation. The idea is to associate a continuum $p$-form with a

\footnotetext{
${ }^{1} \Gamma$ and $K$ commute on the $\lambda=0$ subspace and hence zero modes can be labeled with a given eigenvalue of $\Gamma$.

${ }^{2}$ In flat space it is easy to see that $\Gamma$ acts like an axial rotation on the spinors corresponding to columns of the matrix $\Psi$.
} 
$p$-cochain which is a real or complex linear function over $p$-simplices, $f\left(C_{p}\right)$, where an individual $p$-simplex is denoted $C_{p}[7,8]$. The operators $d$ and $d^{\dagger}$ have corresponding discrete analogs in terms of so-called co-boundary, $\bar{\partial}$, and boundary, $\partial$, operators that act on such $p$-simplices. For example the boundary operator $\partial$ acts on a $p$-simplex consisting of the (ordered) vertices $\left[a_{0}, \ldots, a_{p}\right]$ as

$$
\partial\left[a_{0}, \ldots, a_{p}\right]=\sum_{i}(-1)^{i}\left[a_{0}, \ldots, \hat{a}_{i}, \ldots, a_{p}\right]
$$

where the hat on vertex $\hat{a}_{i}$ indicates that the vertex is omitted. Thus the operation of $\partial$ on some $p$-simplex yields an oriented sum of $(p-1)$-simplices in its boundary

$$
\partial f\left(C_{p}\right)=\sum_{C_{p-1}} I\left(C_{p}, C_{p-1}\right) f\left(C_{p-1}\right)
$$

where $I\left(C_{p}, C_{p-1}\right)$ is the incidence matrix for the triangulation which takes the value +1 if $C_{p-1}$ is contained in the boundary of $C_{p}$ with the correct orientation, -1 if it is contained in the boundary with the opposite orientation, and is zero otherwise. Similarly the coboundary operator $\bar{\partial}$ is defined by

$$
\bar{\partial} f\left(C_{p}\right)=\sum_{C_{p+1}} I^{T}\left(C_{p}, C_{p+1}\right) f\left(C_{p+1}\right)
$$

From these definitions one can verify that $\partial^{2}=\bar{\partial}^{2}=0$. The discrete Kähler-Dirac operator is defined by

$$
K_{D}=\partial-\bar{\partial}
$$

which squares to the discrete Laplacian. Furthermore it should be clear that $\left\{\Gamma, K_{D}\right\}=0$ when $\Gamma$ acts on $p$-cochains instead of $p$-forms and therefore there is still an exact $\mathrm{U}(1)$ symmetry present just as for the continuum theory, acting now on $p$-cochains as

$$
\omega_{p} \rightarrow e^{i \theta(-1)^{p}} \omega_{p}
$$

For any given triangulation the fermion measure is just

$$
\prod_{i_{0}=1}^{N_{0}} d \omega_{i_{0}} d \bar{\omega}_{i_{0}} \prod_{i_{1}=1}^{N_{1}} d \omega_{i_{1}} d \bar{\omega}_{i_{1}} \ldots \prod_{i_{D}=1}^{N_{D}} d \omega_{i_{D}} d \bar{\omega}_{i_{D}}
$$

where $N_{p}$ is the number of $p$-simplices in the triangulation and the indices $i_{p}$ run from $1 \ldots N_{p}$. The $\omega_{i_{p}}$ is the fermion field associated with the $\mathrm{i}^{\text {th }} p$-simplex. Under the $\mathrm{U}(1)$ symmetry in eq. (3.5) this measure transforms by the phase factor $e^{i 2 \theta \chi}$ where the Euler character $\chi$ is given by $[9]$

$$
\chi=N_{0}-N_{1}+\ldots+(-1)^{p} N_{p}
$$

If $\chi \neq 0$ this breaks the $\mathrm{U}(1)$ down to $Z_{2 \chi}$, which depends only on the Euler characteristic and is otherwise independent of the triangulation. 
Since the non-zero eigenvalues of the lattice operator $K_{D}$ again come in complex conjugate pairs this phase must arise from the zero modes of the discrete Kähler-Dirac operator, which must again be either even or odd under $\Gamma$. Homology theory guarantees that such exact zero modes exist even in the discrete system and are simultaneously zero modes of the discrete Laplacian

$$
\square=-\partial \bar{\partial}-\bar{\partial} \partial=-\sum_{C_{p-1}} I\left(C_{p}, C_{p-1}\right) I^{T}\left(C_{p-1}, C_{p}\right)-\sum_{C_{p+1}} I^{T}\left(C_{p}, C_{p+1}\right) I\left(C_{p+1}, C_{p}\right) .
$$

By the Hodge theorem (which applies equally to both discrete and continuum theories) we know that $n_{+}-n_{-}=\chi$, which is consistent with the previous computation of the phase change of the measure. Notice that the anomaly vanishes in odd dimensions since $\chi=0$ there and $n_{+}=n_{-}$.

\section{Condensates}

From this point we confine our discussion to the sphere $S^{D}$ with $\chi=2$ although it is trivial to generalize to arbitrary Euler characteristic. In this case there are precisely 2 zero modes of the Laplacian associated with the 0 and $D$-forms (co-chains) both of which have eigenvalues of $\Gamma$ equal to one.

Let us now consider the partition function on a discrete triangulation $T$ of the sphere with $N_{D} D$-simplices and $N_{0}$ vertices in the presence of fermionic sources $\eta, \bar{\eta}$ of KählerDirac type, paying careful attention to the would-be zero modes that arise in the massless limit

$$
Z(\eta, \bar{\eta})=\operatorname{det}\left(K_{D}^{\prime}(T)+m\right) e^{\left[\bar{\eta}\left(K_{D}^{\prime}(T)+m\right)^{-1} \eta\right]} \times m^{2} e^{\sum_{i=1}^{2} \frac{1}{m} \bar{\eta}_{0}^{i} \eta_{0}^{i}}
$$

where the prime denotes that zero modes satisfying $\square \Phi_{0}^{i}=0$ have been omitted and

$$
\eta_{0}^{i}=\left[\eta \bar{\Phi}_{0}^{i}\right] \quad \bar{\eta}_{0}^{i}=\left[\bar{\eta} \Phi_{0}^{i}\right]
$$

corresponds to the $\mathrm{i}^{\text {th }}$ zero mode component of the source. Notice that the first non-zero contribution to $Z$ as $m \rightarrow 0$ occurs by expanding the zero mode sources to quadratic order and yields

$$
Z(\eta, \bar{\eta})=\operatorname{det}\left(K_{D}^{\prime}(T)+m\right) e^{\left[\bar{\eta}\left(K_{D}^{\prime}(T)+m\right)^{-1} \eta\right]} \bar{\eta}_{0}^{1} \eta_{0}^{1} \bar{\eta}_{0}^{2} \eta_{0}^{2} .
$$

The structure of this expression resembles the usual 't Hooft vertex seen in QCD [10] and generates a non-local four fermion interaction in the theory. Differentiating with respect to the sources will yield a non-zero four fermion correlation function that survives the $m=0$ limit and breaks the $\mathrm{U}(1)$ symmetry down to $Z_{4}$

$$
\langle\bar{\Omega}(x) \Omega(y) \bar{\Omega}(z) \Omega(w)\rangle=\frac{1}{Z} \frac{\delta}{\delta \eta(x)} \frac{\delta}{\delta \bar{\eta}(y)} \frac{\delta}{\delta \eta(z)} \frac{\delta}{\delta \bar{\eta}(w)} Z
$$

where $x, y, z, w$ range over the discrete set of coordinates on the triangulation. To compute this correlator we consider a triangulation in which all simplices are equilateral with fixed 
side length. The discrete Laplacian is block diagonal in the $p$-forms. The 0 -simplex block matrix follows from eq. (3.8) and takes the form

$$
-\square_{i j}=q_{i} \delta_{i j}-C_{i j}
$$

where $C_{i j}=1$ if the vertex $j$ is neighbor to vertex $i$ and $q_{i}$ is the total number of neighbor vertices. The first zero mode solution then corresponds to a vector with constant non-zero 0-form components only

$$
\Phi_{0}^{p}=A \delta^{p 0}(1,1, \ldots) .
$$

Normalizing this vector to unity yields $A=1 / \sqrt{N_{0}}$. The second zero mode is gotten from the $D$-simplex block of the Laplacian and has a structure similar to that given in eq. (4.5) with the matrix element $C_{i j}$ non-zero for each face separating simplices and $q_{i}=(D+1)$ since each $D$-simplex possesses $(D+1)$ neighbors. The corresponding zero mode is

$$
\Phi_{0}^{p}=\frac{1}{\sqrt{N_{D}}} \delta^{p D}(1,1, \ldots) .
$$

Inserting these results into eq. (4.4) shows that the correlator in eq. (4.4) becomes

$$
\left\langle\bar{\omega}_{0}(x) \omega_{0}(y) \bar{\omega}_{D}(z) \omega_{D}(w)\right\rangle=\frac{1}{N_{0} N_{D}} .
$$

Using Wick's theorem this can be written as the product of two-point functions

$$
\left\langle\bar{\omega}_{0}(x) \omega_{0}(y)\right\rangle\left\langle\bar{\omega}_{D}(z) \omega_{D}(w)\right\rangle
$$

where correlators like $\left\langle\bar{\omega}_{0}(x) \omega_{D}(z)\right\rangle$ and $\left\langle\bar{\omega}_{0}(x) \bar{\omega}_{D}(z)\right\rangle$ vanish since the inverse fermion operator can be written as $(-K+m) /\left(-\square+m^{2}\right)$ which only couples $p$-cochain fields to $p$ or $p \pm 1$ co-chains. Furthermore, by translation invariance these two point functions depend only on the difference of their spacetime arguments and since the r.h.s. of eq. (4.8) is independent of coordinates this implies the presence of two bilinear condensates spontaneously breaking $Z_{4}$ to $Z_{2}$

$$
\begin{aligned}
\left\langle\bar{\omega}_{0}(x) \omega_{0}(x)\right\rangle & =\frac{1}{N_{0}} \\
\left\langle\bar{\omega}_{D}(x) \omega_{D}(x)\right\rangle & =\frac{1}{N_{D}}
\end{aligned}
$$

These lattice expressions can be expressed in physical units by writing $N_{0}$ and $N_{D}$ in terms of the physical volume $V$ and the cut-off $a$. For example the $D$-simplex condensate is given by $\frac{1}{a^{D-1}}\left(V / a^{D}\right)^{-1}$. Note that the magnitude of this condensate depends only on the volume of the space and the Euler number and is independent of the triangulation or continuum metric.

\section{$5 \quad$ Numerical results}

We can illustrate this effect using results obtained from an ensemble of random triangulations of the two sphere corresponding to the partition function

$$
Z=\sum_{T} e^{-S(T)}
$$




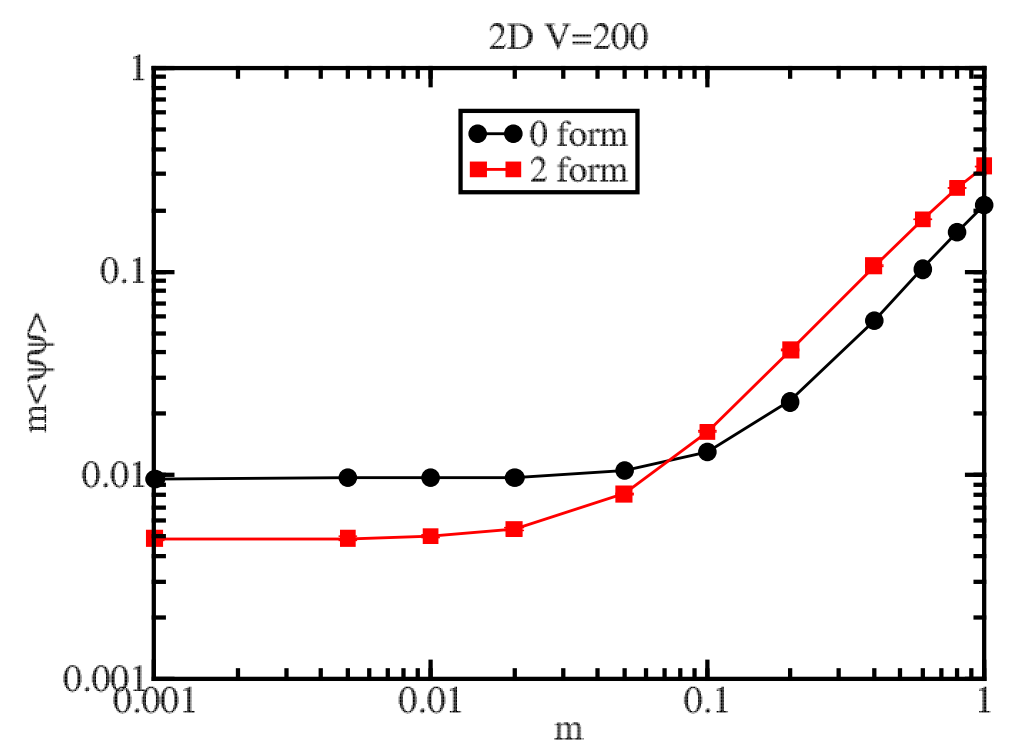

Figure 1. Bilinear condensates vs bare mass $m$ on $S^{2}$ with $N_{2}=200$ and $\beta=0$.

where the action

$$
S(T)=-\beta \sum_{i}^{N_{0}} \log q_{i}+\kappa_{2} N_{2}
$$

This action contains both a bare cosmological constant $\kappa_{2}$ and a coupling $\beta$ that controls the local connectivity of the triangulation.

The code we use is described in [9] and generates an ensemble of so-called combinatorial triangulations in which each simplex is uniquely specified by its vertices. These differ from the degenerate triangulations employed in, for example, [6] although none of our results depend on this distinction. The code uses a Metropolis algorithm to sample the space of triangulations using a set of ergodic local changes to the triangulation described in [9]. We generate ensembles of 10000 configurations with each configuration separated by 10 sweeps (the average acceptance rate of the algorithm is 10\%). Errors are assessed by binning the data in the standard way and looking for stability as the bin size is varied.

We show in figure 1 both the 0 and 2-form bilinear condensates as a function of the bare mass $m$ (the 1-form condensate vanishes as expected for $m \rightarrow 0$ ). Notice that the anomaly generated condensate in the theory with dynamical Kähler-Dirac fermions is extracted by multiplying the condensate measured in the quenched ensembles by the fermion mass. Hence we show $m\langle\bar{\psi} \psi\rangle$ in our plots and tables. We see that the observed value matches very well with theoretical expectations. The volume dependence of these condensates at $\beta=0$ and $m=0.001$ is shown in figure 2 and shows the expected $1 / V$ behavior.

We can further explore the topological character of these condensates by varying $\beta$, which changes the types of triangulation that dominate $Z$ but does not change the global topology. Table 1 shows the condensates for $\beta=-4.0,0.0,4.0$ at $m=0.001$ and $N_{2}=200$.

It should be clear that the magnitude of the condensates do not even depend on the dimensionality of the triangulation. Simulations of the four sphere (see table 2) yield the same condensates taking into account the change in $N_{0}$ with $\beta$ (in two dimensions $\left.2\left(N_{0}-2\right)=N_{2}\right)$. 


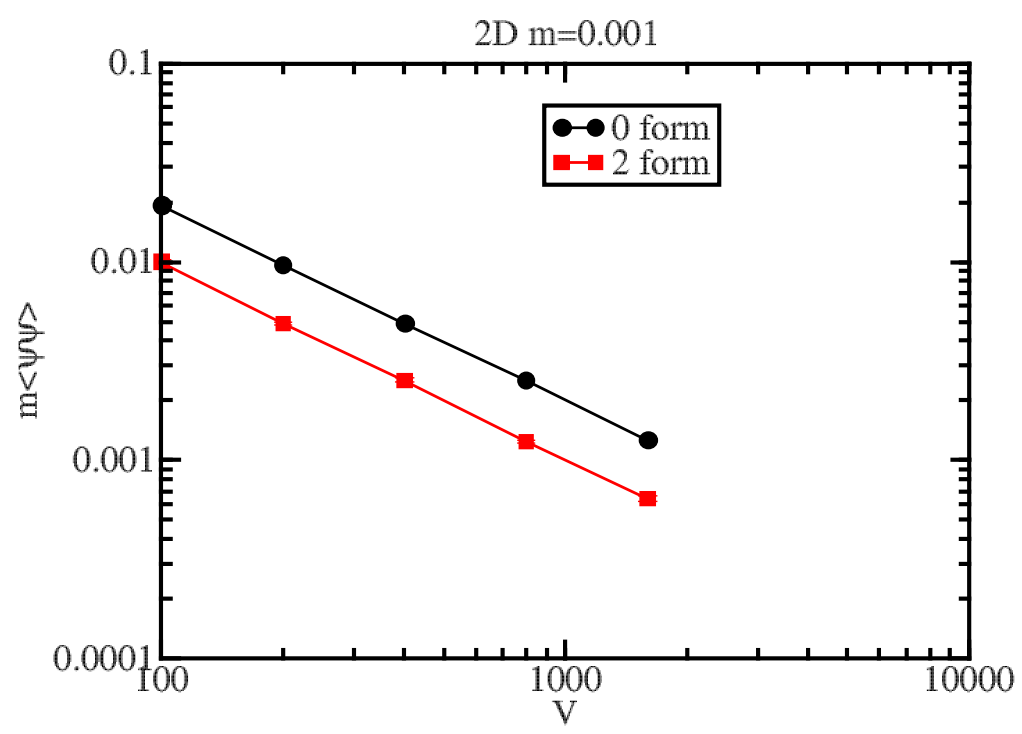

Figure 2. Bilinear condensates vs volume on $S^{2}$ with $m=0.001$ and $\beta=0$.

\begin{tabular}{|c|c|c|c|}
\hline$\cdot$ & $\beta=4.0$ & $\beta=0.0$ & $\beta=-4.0$ \\
\hline$m\left\langle\bar{\omega}_{0} \omega_{0}\right\rangle$ & $0.0101(2)$ & $0.0096(2)$ & $0.0097(2)$ \\
\hline$m\left\langle\bar{\omega}_{2} \omega_{2}\right\rangle$ & $0.00491(7)$ & $0.00490(9)$ & $0.00476(8)$ \\
\hline
\end{tabular}

Table 1. Condensates for several values of the measure coupling $\beta$ for $S^{2}$ using $m=0.001$ and $N_{2}=200, N_{0}=102$.

\begin{tabular}{|c|c|c|c|}
\hline$\cdot$ & $\beta=4.0$ & $\beta=0.0$ & $\beta=-4.0$ \\
\hline$m\left\langle\bar{\omega}_{0} \omega_{0}\right\rangle$ & $0.0186(3)$ & $0.034(1)$ & $0.053(1)$ \\
\hline$m\left\langle\bar{\omega}_{4} \omega_{4}\right\rangle$ & $0.0050(1)$ & $0.0050(1)$ & $0.0051(1)$ \\
\hline $1 / N_{0}$ & $0.0187(1)$ & $0.0341(1)$ & $0.0527(1)$ \\
\hline
\end{tabular}

Table 2. Dependence of condensates on measure coupling $\beta$ for $S^{4}$ using $N_{4}=200$ and bare mass $m=0.001$.

\section{Conclusions}

In this paper we have discussed an anomaly that arises in a theory of Kähler-Dirac fermions formulated on a compact manifold. While anomalies are usually thought of as arising only in continuum theories, in this case the anomaly survives intact in a discretized version of the theory. The reason is simple; the anomaly is sensitive only to the topology of the background space which can be captured exactly in the lattice theory as a result of the precise correspondence between the theory of differential forms and the theory of cochains - the subject of homology theory. As in Fujikawa's derivation of the chiral anomaly, this topological anomaly arises from the non-invariance of the fermion measure under a particular U(1) symmetry specific to Kähler-Dirac fermions. 
We have explained in some detail this correspondence and how the discrete KählerDirac operator is constructed on a triangulation. We should add that our work constitutes the first numerical study of Kähler-Dirac fermions on random simplicial lattices. In the context of dynamical triangulation models of quantum gravity the inclusion of Kähler-Dirac fermions can potentially play a crucial role in determining both the phase structure of the system and the nature of the typical geometries that dominate the partition function. We hope to examine some of these issues in upcoming work [11]. Here, however, we would like to stress that the anomaly, being topological in character, is completely agnostic about the properties of background space or the nature of any gravitational fluctuations - the effect we describe will be true for a wide range of gravitationally coupled theories involving Kähler-Dirac fermions in regimes described by both classical and quantum gravity both on and off the lattice.

It is interesting to ask whether these results could have any implication for cosmology. Since Kähler-Dirac fermions behave locally like multiple copies of Dirac fermions in regions where the curvature is small they are not necessarily ruled out by experiment. Of course there are also global differences between Kähler-Dirac fermions and Dirac fermions. Unlike Dirac fermions, Kähler-Dirac fermions typically possess zero modes even on positive curvature manifolds which is the origin of the anomaly discussed in this paper. ${ }^{3}$ The physical manifestation of this anomaly is the existence of a fermion condensate. The magnitude of the latter is set by the Planck scale times the inverse volume of the Universe in Planckian units. Presumably such a condensate would feed into the dynamics of the Universe at early times and might have implications for cosmology.

\section{Acknowledgments}

This work is supported in part by the U.S. Department of Energy, Office of Science, Office of High Energy Physics, under Award Number DE-SC0009998. The authors are grateful for discussions with Jay Hubisz.

Open Access. This article is distributed under the terms of the Creative Commons Attribution License (CC-BY 4.0), which permits any use, distribution and reproduction in any medium, provided the original author(s) and source are credited.

\section{References}

[1] T. Banks, Y. Dothan and D. Horn, Geometric fermions, Phys. Lett. B 117 (1982) 413 [INSPIRE].

[2] S. Catterall, D.B. Kaplan and M. Ünsal, Exact lattice supersymmetry, Phys. Rept. 484 (2009) 71 [arXiv:0903.4881] [INSPIRE].

[3] L. Susskind, Lattice Fermions, Phys. Rev. D 16 (1977) 3031 [InSPIRE].

[4] E. Kähler, Der innere Differentialkalkül, Rend. Mat. XXI (1962) 425.

\footnotetext{
${ }^{3}$ This also makes them interesting from the point of view of Kaluza-Klein reduction since it allows for light fermions to appear in the compactified theory.
} 
[5] J. Ambjørn, J. Jurkiewicz and R. Loll, Causal Dynamical Triangulations and the Quest for Quantum Gravity, in Proceedings, Foundations of Space and Time: Reflections on Quantum Gravity, Cape Town, South Africa, pp. 321-337 (2010) [arXiv: 1004.0352] [INSPIRE].

[6] J. Laiho, S. Bassler, D. Coumbe, D. Du and J.T. Neelakanta, Lattice Quantum Gravity and Asymptotic Safety, Phys. Rev. D 96 (2017) 064015 [arXiv: 1604.02745] [InSPIRE].

[7] J.M. Rabin, Homology Theory of Lattice Fermion Doubling, Nucl. Phys. B 201 (1982) 315 [INSPIRE].

[8] P. Becher and H. Joos, The Dirac-Kähler Equation and Fermions on the Lattice, Z. Phys. C 15 (1982) 343 [INSPIRE].

[9] S. Catterall, Simulations of dynamically triangulated gravity: An Algorithm for arbitrary dimension, Comput. Phys. Commun. 87 (1995) 409 [hep-lat/9405026] [INSPIRE].

[10] G. 't Hooft, Symmetry Breaking Through Bell-Jackiw Anomalies, Phys. Rev. Lett. 37 (1976) 8 [inSPIRE].

[11] S. Catterall, J. Laiho and J. Unmuth-Yockey, Kahler-Dirac fermions on Euclidean dynamical triangulations, in preparation. 